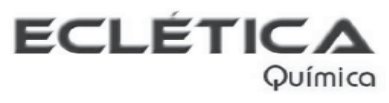

www.scielo.br/eq

Volume 29, número 2, 2004

\title{
Caracterização de ácidos húmicos de diferentes origens por eletroforese capilar de zona
}

\author{
S. L. de Moraes*, M. D. Landgraf*, M. O. O. Rezende* \\ * Instituto de Química de São Carlos, Universidade de São Paulo \\ Av. Trabalhador Sãocarlense , 400, 13560-970 São Carlos-SP \\ e-mail:sollm@ig.com.br
}

\begin{abstract}
Resumo: O objetivo deste trabalho foi caracterizar ácidos húmicos (HA) de diferentes origens por eletroforese capilar de zona (CZE) e avaliar a influência da concentração do tampão borato nesta caracterização. Os resultados mostraram que HA de diferentes origens podem ser rapidamente caracterizados por CZE. Por esta técnica, a caracterização de HA é devido a diferenças na razão carga/ tamanho e das propriedades dos componentes individuais, conseqüência de suas diferentes origens.
\end{abstract}

Palavras-chave: eletroforese capilar; ácidos húmicos; caracterização.

\section{Introdução}

Ácidos húmicos (HA) são compostos de coloração escura e poliméricos, formados pela associação de macromoléculas heterogêneas contendo grupos polifuncionais de alto peso molecular [1]. Por se tratar de uma matriz complexa que varia em composição, dependendo da sua origem (animal, vegetal e microbiológica) a estrutura dos HA ainda não é totalmente elucidada [2]. A ordem de ionização dos HA é governada principalmente pela quantidade de grupos fenólicos e carboxílicos ionizáveis, a qual é função do $\mathrm{pH}[2,3]$.

Devido a suas características estruturais, os HA podem interagir com metais e compostos orgânicos como, por exemplo, pesticidas presentes nos compartimentos ambientais. Por isso, é importante o desenvolvimento de novos métodos analíticos que auxiliem na compreensão do comportamento dos HA no ambiente $[4,5]$.

Atualmente, aumentou-se o interesse pela aplicação da eletroforese capilar de zona (CZE) na separação e caracterização de HA, principalmente devido à natureza iônica dessas moléculas e à facilidade de utilização desse modo de eletroforese [6-11]. Em CZE, a separação ocorre devido à migração diferencial de compostos iônicos ou ionizáveis em um campo elétrico, a qual é função da razão carga e tamanho do íon e da presença do fluxo eletroosmótico (EOF) para um dado $\mathrm{pH}$ [12]. As vantagens de utilização desta técnica são: rápida capacidade de separação, tempo de análise relativamente curto, facilidade de operação e automação, vários modos de operação, alto poder de resolução, injeção de pequeno volume de amostra e exposição mínima do analista a substâncias químicas tóxicas [12,13].

Muitos trabalhos têm sido propostos para a caracterização de HA por CZE [5-8]. No entanto, no Brasil estes estudos ainda são muito pouco difundidos. Dessa forma, o objetivo deste trabalho foi caracterizar ácidos húmicos de diferentes origens por CZE e avaliar o efeito da concentração do tampão borato nesta caracterização, uma vez que muitos autores afirmam que os HA podem formar complexos com os íons borato, dificultando 
a interpretação dos eletroferogramas dos HA $[7,14,15]$.

\section{Material e Métodos \\ Amostragem}

As amostras de ácidos húmicos (HA) utilizadas neste trabalho foram: HA de turfa (THA) coletadas a uma profundidade de 10 a $25 \mathrm{~cm}$ em uma turfeira às margens do rio Mogi-Guaçu, localizada nas proximidades do município de Luiz Antônio, no estado de São Paulo, HA de vermicomposto (VHA) produzida a partir do esterco bovino e tratada com Eisenia foetida ou Lumbriculus rubellus e HA comercial da Aldrich (AHA) lote $n^{0} 05924 \mathrm{KN}$.

\section{Reagentes e soluções}

Todos os reagentes utilizados foram de padrão analíticos adquiridos pela Merck ou Aldrich. As soluções foram preparadas com água purificada pelo sistema milli-Q (Millipore). A solução tampão utilizada foi $20 \mathrm{mmol} / \mathrm{L}$ borato $\left(\mathrm{H}_{3} \mathrm{BO}_{3}+\mathrm{NaOH}\right) \mathrm{pH} 9,3$.

As amostras de HA foram dissolvidas em uma pequena quantidade de $\mathrm{NaOH} 0,1 \mathrm{~mol} / \mathrm{L}$ e o volume completado com água deionizada para obter a concentração de 1mg/mL de HA.

Extração, purificação e determinação da acidez do ácido húmico

A extração e purificação do HA foram realizadas segundo o procedimento adaptado da Sociedade Internacional de Substâncias Húmicas [16], baseado nas extrações ácido-base seguido de purificação através de sucessivas eluições em resinas de troca iônica.

Para a determinação de acidez de HA utilizou-se o método desenvolvido por Schnitzer e Gupa, em 1965 [17]. A titulação da acidez total foi seguida em um sistema modificado por Prado et.al [18], para evitar a formação de carbonatos pela presença de $\mathrm{CO}_{2}$.

\section{Efeito da concentração do tampão borato na caracterização do HA \\ O Efeito da concentração do tampão borato} na caracterização de HA por CZE foi estudado preparando-se uma solução estoque de $100 \mathrm{mmol} /$ L de borato de sódio. A partir de diluições da solução estoque prepararam-se as demais concentrações: 10; 20; 30; 40 e $50 \mathrm{mmol} / \mathrm{L}$ para pH 9,3.

\section{Condições eletroforéticas}

Para os experimentos em eletroforese capilar (CE) utilizou-se um sistema $\mathrm{HP}{ }^{3 \mathrm{D}} \mathrm{CE}$ (Hewlett Packard, USA) com um capilar de 64,5 cm de comprimento total, $56 \mathrm{~cm}$ de comprimento efetivo e $50 \mathrm{~mm}$ de diâmetro interno. A detecção foi feita "on column" pelo sistema de UV-VIS com arranjos de diodo. Para todos os experimentos em CZE o comprimento de onda utilizado foi $214 \mathrm{~nm}$. As condições de análise por CZE foram: $20 \mathrm{mmol} /$ L de tampão borato, $\mathrm{pH}$ 9,3; injeção hidrodinâmica (50 mbar por 10s); tensão: $20 \mathrm{kV}$; temperatura: $30{ }^{\circ} \mathrm{C}$.

O condicionamento do capilar foi realizado antes das corridas por lavagem com as seguintes soluções: 5 minutos com $\mathrm{NaOH} 1 \mathrm{~mol} / \mathrm{L}, 5$ minutos com $\mathrm{NaOH}$ 0,1 mol/L, 5 minutos com água e 15 minutos com o tampão de corrida. Entre cada corrida o capilar foi lavado com $\mathrm{NaOH} 1 \mathrm{~mol} / \mathrm{L}$ durante 1 minuto, água ultrapura (millipore) por 1 minuto e com 3 minutos com solução tampão.

\section{Resultados e discussão}

Atenção especial tem sido dada à interpretação dos eletroferogramas dependendo do sistema de tampão utilizado. Por exemplo, tampão borato pode formar complexos com os HA gerando picos adicionais de HA-borato em função do $\mathrm{pH}$ e da concentração dos íons borato [7]. A Figura 1 apresenta o perfil de separação em função da concentração do tampão borato para o THA.

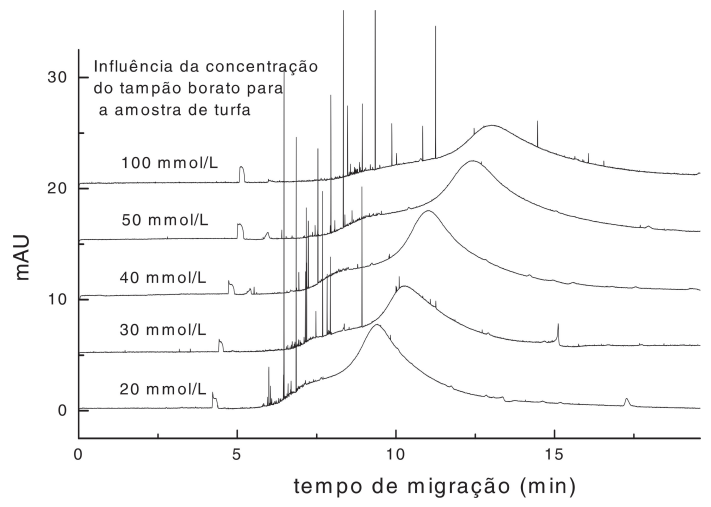

Figura 1- Variação da concentração de tampão borato $\mathrm{pH} 9,3$ para a amostra de THA (1 mg/mL). Condições: $20 \mathrm{kV}$; injeção hidrodinâmica (50 mbar por $10 \mathrm{~s}$ ), $30^{\circ} \mathrm{C}, \mathrm{L}=64,5 \mathrm{~cm}$ e l $=56 \mathrm{~cm}$, $214 \mathrm{~nm}$. 
Variando-se a concentração de tampão borato não foi observado picos adicionais na banda do THA, somente um deslocamento da banda para maiores concentrações de tampão, o que por ser explicado devido à compressão da dupla camada elétrica levando, neste caso, a um aumento no tempo de migração para maiores concentrações de tampão. A dispersão da banda observada para maiores concentrações de tampão ocorre devido ao THA permanecer mais tempo na coluna. $\mathrm{O}$ comportamento do THA foi similar para todas as concentrações estudadas.

Para averiguar se o surgimento de novos picos dependia da amostra utilizada, trabalhou-se, também, com uma amostra de VHA e não se observou alteração nos picos do HA com a variação da concentração de tampão borato (Figura 2).

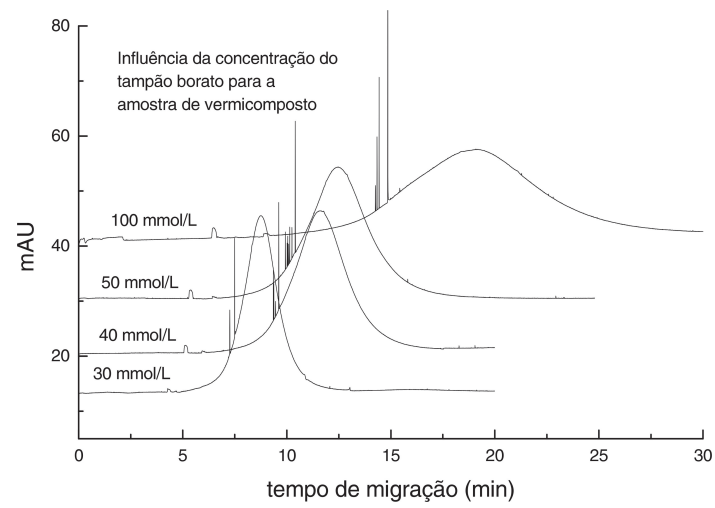

Figura 2- Variação da concentração de tampão borato pH 9,3 para a amostra de VHA (1mg/mL). Condições: $20 \mathrm{kV}$; injeção hidrodinâmica ( 50 mbar por $10 \mathrm{~s}$ ), $30^{\circ} \mathrm{C}, \mathrm{L}=64,5 \mathrm{~cm}$ e l $=56$ $\mathrm{cm}, 214 \mathrm{~nm}$.

Os resultados obtidos sugerem que a formação de complexo de borato com HA não depende da concentração dos íons borato e tampouco da amostra, uma vez que para as amostras de THA e VHA não houve surgimento de picos adicionais nos eletroferogramas.

\section{Caracterização do HA por eletroforese capilar}

O comportamento de HA em CZE depende da razão entre a sua carga e tamanho. Sendo assim, se duas amostras de HA exibem o mesmo comportamento em um campo elétrico, então essas amostras apresentam praticamente a mesma razão carga/tamanho [8,9]. Diferenças entre a mobilidade, intensidade e absorção são estabelecidas pelas diferenças estruturais de HA de diferentes regiões. Portanto, HA de diferentes origens podem ser comparados se a separação é feita nas mesmas condições de análise. Neste contexto, o tempo de migração juntamente com o perfil de separação do HA será um critério de distinção.

A Figura 3 apresenta o eletroferograma dos HA analisados por CZE. Nas condições eletroforéticas estudadas a maioria dos grupos carboxílicos e fenólicos presentes na molécula do HA encontram-se na forma desprotonada e migram como ânions.

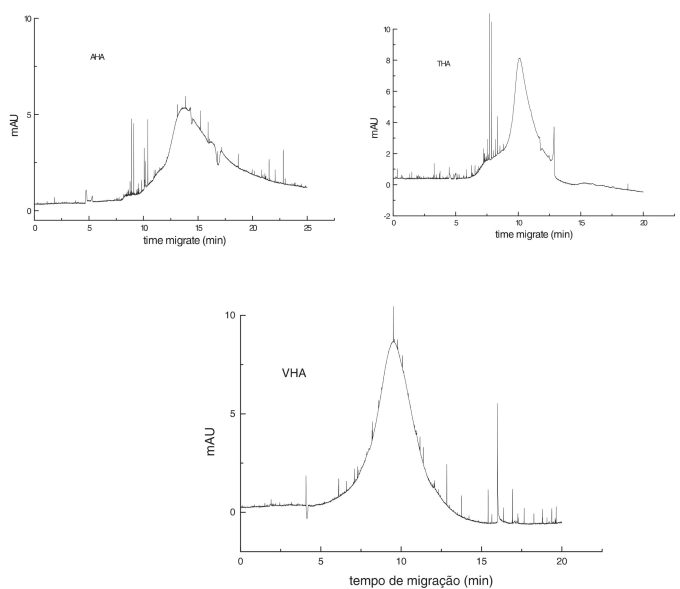

Figura 3- Eletroferogramas dos HA (1mg/mL) analisados por CZE. Condições: $20 \mathrm{mmol} / \mathrm{L}$ de tampão borato, pH 9,3, injeção hidrodinâmica (50 mbar por 10s), 20 kV, $30^{\circ} \mathrm{C}$.

Comparando os eletroferogramas pode-se observar uma banda larga comum a todos os eletroferogramas, a qual é designada por muitos autores como região aniônica (região em que se encontra a maioria dos grupos carboxílicos e fenólicos) [7-10,19]. Essa banda larga é devida ao fato dos HA apresentarem mobilidades muito próximas não havendo distinção na dissociação dos vários grupos funcionais presentes na molécula de HA.

Analisando cada perfil eletroforético e considerando que a medida de ionização dessas macromoléculas é governada principalmente pela quantidade de grupos carboxílicos e fenólicos, em função do pH da solução e que a mobilidade de um íon é função da relação carga/tamanho desse íon, pode-se verificar uma maior mobilidade aparente média para o VHA (menor tempo de 
migração). Isto pode ser interpretado como menor razão carga/tamanho e menor acidez total para essa molécula. Já, para o AHA a mobilidade aparente média é menor (maior tempo de migração), sendo assim, uma maior razão carga/tamanho e, consequentemente, uma maior acidez total é observada para o AHA do que para o THA e VHA. Essa observação coincide com os experimentos de acidez total apresentados na Tabela 1.

\section{Conclusão}

A CZE apresentou-se como uma ferramenta poderosa na caracterização de HA, uma vez que vários HA de diferentes origens podem ser rapidamente caracterizados. Pelo uso do tampão apropriado, os eletroferogramas podem ser comparados e interpretados em termos de mudanças nas características estruturais dos HA. Uma das principais vantagens ao se utilizar à eletroforese capilar é a capacidade de trabalhar com pequenas quantidades de amostra. O comportamento dos HA por CZE reforça um modelo supramolecular para a estrutura do HA.

Agradecimentos

As autoras agradecem à FAPESP e ao CNPq pelo apoio financeiro. Solange L. de Moraes agradece à FAPESP (processo \# 00/02368-5) pela bolsa de doutorado concedida.

Recebido em 14/02/04 Aceito em 24/05/04

TABELA 1- Características dos HA de estudados.

\begin{tabular}{|l|l|l|l|}
\hline Determinações & THA & VHA & AHA \\
\hline acidez carboxílica $(\mathrm{mmol} \mathrm{H+/g)}$ & 2,76 & 0,06 & 3,91 \\
\hline acidez fenólica $(\mathrm{mmol} \mathrm{H+/g)}$ & 1,39 & 3,94 & 2,93 \\
\hline acidez total $(\mathrm{mmol} \mathrm{H+/g} \mathrm{de} \mathrm{HA)}$ & 4,15 & 4,00 & 6,84 \\
\hline
\end{tabular}

S. L. de Moraes, M. D. Landgraf, M. O. O. Rezende. Characterization of humic acids of different origins by capillary electrophoresis.

Abstract: The purpose of this work was characterization humic acids (HA) of different origins by capillary zone electrophoresis (CZE). The influence of the concentration of borate in the characterization was investigated. The results show the potential of CZE for the characterization of different HA samples, thus HA of different origins can be rapidly characterized. The characterization of HA are due to difference charge-to-size ratios and the various structural properties of the individual components caused by their different origins.

Keywords: capillary electrophoresis; humic acids; characterization.

\section{Referências}

[1] A. P. P. Toledo, A. M. L. Neptune, Trop. Ecol. 5 (1994) 248.

[2] A.Piccolo, P.Conte, Advances Environ. Res. 3 (2000) 508.

[3] W.T.L. Silva, C. Thobie-Gautier, M.O.O.

Rezende, N. El Murr, Electroanalysis 14 (2002) 71.

[4] S.L. Moraes, M.O.O. Rezende, Pesticidas: R. Ecotox. e Meio Ambiente. 8 (1998)157.
[5] A.H. Rosa, J.C. Rocha, M. Furlan, Quim. Nova 23 (2000) (4) 472.

[6]M. L. Pacheco, J. Havel, Electrophoresis 23 (2002) 268.

[7] D. Fetsch, J. Havel, J. Chromatogr. A 802 (1998) 189.

[8] D. Fetsch, M. Hradilová, E. M. P. Havel, J. Méndez, J. Chromatogr. A 817 (1998) 313. 
[9] S. Pompe, K. Heise, H. Nitsche, J. Chromatogr. A 723 (1996) 215.

[10] L. Pokorná, M. L. Pacheco, J. Havel, J. Chromatogr. A 895 (2000) 345.

[11] Ph. Schmitt-Kopplin, A. W. Garrison, E. M. Perdue, D. Freitag, A. Kettrup,

J.Chromatogr. A 807 (1998)101.

[12] H.J. Issaq, Electrophoresis, 21 (2000) 1921.

[13] K. D. Altria, J.Chromatogr. A 856 (1999)

443.

[14] Ph. Schmitt-Kopplin, N. Hertkorn, A. W. Garrison, A. Kettrup, Anal. Chem. 70 (1998) 3798.
[15] R. Dunkelong, H. Rüttinger, K. Peisker, J. Chromatogr. A 777 (1997) 355.

[16] Schnitzer, M. Soil Organic Matter; Schnitzer, M.; Khan, S. U., Amsterdam: Elsevier, 1978, p. 1-64.

[17] M. Schintzer, U. C. Gupta, Soil Sci. Soc. Proc. 29 (1965) 274.

[18] A. G. S. Prado, S. M. Souza, W. T. L. Silva, M. O. O. Rezende, Quim. Nova 22(6) (1999) 894. [19] M. D. Landgraf, R. C. Javaroni, M. O. O Rezende, J. Capillary Electrophoresis 5(5) (1998) 193. 